\title{
Initial CT features of COVID-19 predicting clinical category
}

\author{
Li Fan $^{1} \cdot$ Wenqing Le ${ }^{2} \cdot$ Qin Zou ${ }^{1} \cdot$ Xiuxiu Zhou ${ }^{1} \cdot$ Yun Wang ${ }^{1} \cdot$ Hao Tang $^{3,4} \cdot$ Jiafa Han $^{5} \cdot$ Shiyuan Liu $^{1}$
}

Received: 26 March 2020 / Revised: 27 January 2021 / Accepted: 3 February 2021 / Published online: 21 February 2021

(c) The Author(s), under exclusive licence to Springer Nature Singapore Pte Ltd. part of Springer Nature 2021

\begin{abstract}
Purpose To analyze the initial CT features of different clinical categories of COVID-19.

Material and methods A total of 86 patients with COVID-19 were analyzed, including the clinical, laboratory and imaging features. The following imaging features were analyzed, the lesion amount, location, density, lung nodule, halo sign, reversed-halo sign, distribution pattern, inner structures and changes of adjacent structures. Chi-square test, Fisher's exact test, or Mann-Whitney $U$ test was used for the enumeration data. Binary logistic regression analysis was performed to draw a regression equation to estimate the likelihood of severe and critical category. The forward conditional method was employed for variable selection.

Results Significant statistical differences were found in age $(p=0.001)$ and sex $(p=0.028)$ between mild and moderate and severe and critical category. No significant difference was found in clinical symptoms and WBC count between the two groups. The majority of cases (91.8\%) showed multifocal lesions. The presence of GGO was higher in severe and critical category than in the mild and moderate category. $(57.8 \%$ vs.31.7\%, $p=0.015)$. Lymphocyte count was important indicator for the severe and critical category.

Conclusion The initial CT features of the different clinical category overlapped. Combining with laboratory test, especially the lymphocyte count, could help to predict the severity of COVID-19.
\end{abstract}

Keywords COVID-19 $\cdot$ Imaging findings $\cdot$ Clinical category

Li Fan and Wenqing Le contributed equally to the article.

Hao Tang

tanghao_0921@126.com

Jiafa Han

781571340@qq.com

$\triangle$ Shiyuan Liu

liushiyuan@smmu.edu.cn

1 Department of Radiology, Changzheng Hospital, Naval Medical University, No. 415 Fengyang Road, Shanghai 200003, China

2 Department of Critical Care, Wuhan Hankou Hospital, Er Qi Side, Road No. 7, Hubei 430010, China

3 Department of Respiratory and Critical Care Medicine, Changzheng Hospital, Naval Medical University, No. 415 Fengyang Road, Shanghai 200003, China

4 Department of Critical Care, Wuhan Huoshenshan Hospital, Hubei 430100, China

5 Department of Radiology, Wuhan Hankou Hospital, Er Qi Side Road, No. 7, Hubei 430010, China

\section{Introduction}

Since the outbreak of COVID-19 in the late December, 2019. It has been outbroken in 184 countries with 267,013 confirmed cases and 11,201 deaths according to the WHO reports until Mar 21, 2020 [1]. COVID-19 has become a global health crisis due to the high contagion. With the recognition of COVID-19, 7 Trial version of diagnosis and treatment of COVID-19 has been issued by the National Health Commission of the People's Republic of China [2]. COVID-19 is classified into four categories, mild, moderate, severe and critical stage based on the severity [3]. It has been reported about the clinical and imaging features of COVID-19. The initial CT features in different stages may predict the progress and prognosis. The purpose was to evaluate the difference of initial CT features between different stages, then evaluate the predictive value of initial CT features combining the clinical information. 


\section{Material and methods}

\section{Patient population}

From 2020.01.12 to 2020.02.08, a total of 87 patients from Hankou Hospital, Wuhan, China with positive reverse transcription-polymerase chain reaction (RT-PCR) results of COVID-19 were included in this study retrospectively. It was approved by both ethic committees of Hankou hospital (HKYY-2020-023) and Changzheng Hospital (2020SL006), which waived informed consent. One patient was excluded due to the loss of laboratory tests. Therefore, the remained 86 patients were analyzed. Clinical and laboratory information including fever, respiratory symptoms, White blood Cell (WBC) count, lymphocyte count and prognosis were recorded. WBC and lymphocyte count were classified as normal and decreased.

\section{Clinical category}

According to the Protocols-Ver7 [2], clinical stages of COVID-19 is divided into mild type, moderate type, severe type and critical type based on its severity. (1) Mild type, clinical symptoms are mild, and no pneumonia appearance was found in imaging; (2) moderate type, with fever, respiratory symptom or others, and pneumonia appearance on CT images was found; (3) severe type, with any of the following signs, respiratory distress, $\mathrm{RR} \geq 30$ times/min; oxygen saturation $\leq 93 \%$ in resting state; arterial partial pressure of oxygen $(\mathrm{PaO} 2) /$ concentration of oxygen $(\mathrm{FiO} 2) \leq 300 \mathrm{mmHg}$; fast progression of imaging findings (more than $50 \%$ ) within 24-48 h; (4) critical type, with any of the following signs, respiratory failure, mechanical ventilation, shock and other organ failure resulting in the treatment in the Intensive Care Unit (ICU). In this study, the mild and moderate category were merged into one group; and severe and critical category were merged into the other group.

\section{CT imaging analysis}

All the participants underwent imaging on a Siemens Definition AS + 128 (Siemens Healthcare). Breath-hold training was performed before CT scanning. All the scans were noncontrast enhanced scan and performed at the end of inspiration as long as possible. The following imaging parameters were used: $120 \mathrm{kVp}$ /automatic mAs, slice thickness $1 \mathrm{~mm}$, slice interval $1.5 \mathrm{~mm}$, matrix of $512 \times 512$, collimation $128 \times 0.6 \mathrm{~mm}$, rotation $0.5 \mathrm{~s}$, pitch 1.2 and standard resolution algorithms.

All images were evaluated by two chest radiologists and blinded to the clinical categories. The following imaging features were analyzed, the lesion amount (focal or multifocal), location (five lobes), density (ground glass opacity, GGO; consolidation component; and the combination of them), lung nodule, halo sign, reversed-halo sign, distribution pattern (cone-shaped or triangle, subsegmental, and segmental), inner structures (fibrosis, thickening of intralobular septum, 'crazy-paving' sign, thickening of small vessels, air bronchogram, thickening of bronchovascular bundle, and cavity) and changes of adjacent structures including the thickening and/or shifting of pleura, sparing subpleural region, pleural effusion and lymphadenopathy.

\section{Statistical analysis}

The data were analyzed using SPSS22.0 software (SPSS Inc). The data was expressed in mean \pm standard deviation or median (InterQuartile Range, IQR). Chi-square test, Fisher's exact test, or Mann-Whitney $U$ test was used for the enumeration data. A two-sided $p$ value $<0.05$ was considered statistically significant. In addition, binary logistic regression analysis was performed on the clinical and imaging characteristics to draw a regression equation to estimate the likelihood of severe and critical category. The forward conditional method was employed for variable selection. In binary logistic regression analysis, clinical category was regarded as dependent variables (mild and moderate vs. severe and critical), and the demographic data, clinical symptoms, blood test and imaging features were regarded as independent variables. Age older than 60 years was defined as the elder, otherwise set as the non-elder.

\section{Results}

\section{Demographic data, prognosis, clinical symptoms and laboratory tests}

There were 86 confirmed COVID-19 cases (male 40, female 46) with mean age 56 years old (age range 37-76 years old). According to the clinical category, there were 41 with mild and moderate category (mild 2, moderate 39 ) and 45 with severe and critical category (severe 33, critical 12). Significant statistical differences were found in age and sex between mild and moderate and severe and critical category with $p$ value $0.001-0.028$, respectively. The older was more common in the severe and critical category than the mild and moderate category (mean age 63 vs. 49); moreover, the ratio of male to female was higher in the severe and critical category (26:19) than the mild and moderate category (14:27). (Table 1).

Table 1 showed 74 patients $(86 \%)$ were discharged, 9 cases $(10.5 \%)$ were dead and 3 cases $(3.5 \%)$ were transferred to special hospital. The prognosis was significantly different 
Table 1 Demographic data, prognosis, laboratory test and clinical symptoms of patients with COVID-19

\begin{tabular}{lcccr}
\hline & Total $(N=86)$ & $\begin{array}{l}\text { Mild and moderate } \\
(n=41)\end{array}$ & $\begin{array}{l}\text { Severe and critical } \\
(n=45)\end{array}$ & $p$ value \\
\hline Age [median (IQR), year] & $56(37.5-66.8)$ & $49(33-62)$ & $63(50-71)$ & 0.001 \\
Sex & & & & 0.028 \\
$\quad$ Male & $40(46.5 \%)$ & $14(34.1 \%)$ & $26(57.8 \%)$ & \\
Female & $46(53.5 \%)$ & $27(65.9 \%)$ & $19(42.2 \%)$ & \\
Prognosis & & & & \\
Discharge & $74(86.0 \%)$ & $41(100 \%)$ & $33(73.3 \%)$ & $<0.001$ \\
Death & $9(10.5 \%)$ & $0(0 \%)$ & $9(20 \%)$ & 0.003 \\
Transfer & $3(3.5 \%)$ & $0(0 \%)$ & $3(6.7 \%)$ & 0.243 \\
Fever $\left(\geq 37.3{ }^{\circ} \mathrm{C}\right)$ & $77(89.5 \%)$ & $34(82.9 \%)$ & $43(95.6 \%)$ & 0.080 \\
Respiratory symptoms & $64(74.4 \%)$ & $28(68.3 \%)$ & $36(80 \%)$ & 0.214 \\
Normal WBC & $64(74.4 \%)$ & $28(68.3 \%)$ & $36(80 \%)$ & 0.21 \\
Decreased WBC & $14(16.3 \%)$ & $7(17.1 \%)$ & $7(15.6 \%)$ & 0.894 \\
Normal lymphocyte & $31(36.0 \%)$ & $26(17.8 \%)$ & $8(56.1 \%)$ & $<0.001$ \\
Decreased lymphocyte & $52(60.5 \%)$ & $15(36.6 \%)$ & $37(82.2 \%)$ & $<0.001$ \\
Pneumonia on CT & $84(97.6 \%)$ & $39(95.12 \%)$ & $45(100 \%)$ & 0.134 \\
RT-PCR (positive) & $86(100 \%)$ & $41(100 \%)$ & $45(100 \%)$ & N/A \\
\hline
\end{tabular}

Data are median (IQR), $n(\%)$, or $\mathrm{n} / \mathrm{N}(\%)$, where $N$ is the total number of patients with available data. $p$ values comparing common pneumonia and severe, critical pneumonia are from $\chi^{2}$ test, Fisher's exact test, or Mann-Whitney $U$ test. $p<0.05$ has statistically significance between the two groups, higher discharge rate was found in the mild and moderate category.

No significant difference was found in clinical symptoms and WBC count between the two groups. The ratio of decreased lymphocyte count was higher in severe and critical category (37/45) than that in the mild and moderate category (15/41). (Table 1).

\section{Imaging findings}

CT images were evaluated from the lesion amount, location, density, distribution pattern, interface, inner structures and changes of adjacent structures. 2 cases with mild category showed no any abnormal findings. The majority of cases $(91.8 \%)$ showed multifocal lesions with the involvement of 355 lobes in total, predominant in both lower lobes $(n=155)$. The involvement of right upper lobe $(86.7 \%$ vs. $68.3 \%)$ and left upper lobe (93.3\% vs. $82.9 \%$ ) was higher in the severe and critical category than in the mild and moderate category $(p=0.040 ; p=0.022)$. The presence of each imaging feature was listed in Table 2, showing GGO, GGO with consolidation, subsegmental distribution, thickening of intralobular septum, thickening of small vessels, air bronchogram, crazy-paving pattern, thickening of bronchovascular bundles, thickening pleura and shifting of pleura with high presence rate (Figs. 1, 2, 3, 4, 5). Except for GGO, no difference was found in imaging features between the two groups. The presence of GGO was higher in severe and critical category than in the mild and moderate category. $(57.8 \%$ vs.31.7\%, $p=0.015$ ). (Table 2 ).

\section{Binary logistic regression analysis}

The results showed that lymphocyte count was important indicator for the severe and critical category. The odds ratios (ORs) of lymphocyte count were $0.132(p=0.000)$. In other words, the risk of severe and critical category with decreased lymphocyte count was 7.58 times than that with normal lymphocyte count (Supplementary Table).

\section{Discussion}

Imaging plays a great role in the assessment of presence of pneumonia, involvement degree and progression. The diagnosis of suspected COVID-19 should be based on the epidemiological, clinical, laboratory and imaging features. Although the gold criteria are the nucleic acid with RT-PCR, CT can obtain results faster [4]. In present study, we analyzed the clinical and imaging features of different clinical categories and found age, sex, lesion location and the presence of GGO was significantly different between mild and moderate and severe and critical category. Furthermore, lymphocyte count was the important indicator for the severe and critical category by binary logistic regression analysis.

The older and the male were more prone to progress into severe or critical category in comparison with the younger and the female. Wang D [5] found that patients who required ICU care were significantly older (median age, 66 years vs. 51 years; $p<0.001$ ) and were more likely 
Table 2 Imaging features of patients with COVID-19

\begin{tabular}{|c|c|c|c|c|}
\hline Imaging features & Total $(N=86)$ & $\begin{array}{l}\text { Mild and } \\
\text { moderate }(n=41)\end{array}$ & $\begin{array}{l}\text { Severe and } \\
\text { critical }(n=45)\end{array}$ & $p$ value \\
\hline No abnormal & $2(2.3 \%)$ & $2(4.9 \%)$ & $0(0 \%)$ & 0.224 \\
\hline Focal & $7(8.1 \%)$ & $4(9.8 \%)$ & $3(6.7 \%)$ & 0.704 \\
\hline Multifocal & $79(91.8 \%)$ & $35(85.4 \%)$ & $42(93.3 \%)$ & 0.299 \\
\hline RUL & $67(77.9 \%)$ & $28(68.3 \%)$ & $39(86.7 \%)$ & 0.040 \\
\hline RML & $69(80.2 \%)$ & $30(73.2 \%)$ & $39(86.7 \%)$ & 0.116 \\
\hline RLL & $77(89.5 \%)$ & $36(87.8 \%)$ & $41(91.1 \%)$ & 0.731 \\
\hline LUL & $66(76.7 \%)$ & $27(65.9 \%)$ & $39(86.7 \%)$ & 0.022 \\
\hline LLL & $76(88.4 \%)$ & $34(82.9 \%)$ & $42(93.3 \%)$ & 0.183 \\
\hline GGO & $39(45.3 \%)$ & $13(31.7 \%)$ & $26(57.8 \%)$ & 0.015 \\
\hline Consolidation & $22(25.6 \%)$ & $9(22 \%)$ & $13(28.9 \%)$ & 0.461 \\
\hline GGO with consolidation & $41(47.7 \%)$ & $22(53.7 \%)$ & $19(42.2 \%)$ & 0.289 \\
\hline Nodule & $10(11.6 \%)$ & $7(17.1 \%)$ & $3(6.7 \%)$ & 0.183 \\
\hline Halo sign & $0(0 \%)$ & $0(0 \%)$ & $0(0 \%)$ & - \\
\hline Reversed halo sign & $1(1.2 \%)$ & $0(0 \%)$ & $1(2.2 \%)$ & 1.000 \\
\hline Cone-shaped & $35(40.7 \%)$ & $15(36.6 \%)$ & $20(44.4 \%)$ & 0.459 \\
\hline Subsegmental distribution & $75(87.2 \%)$ & $37(90.2 \%)$ & $38(84.4 \%)$ & 0.421 \\
\hline Segmental distribution & $45(52.3 \%)$ & $19(46.3 \%)$ & $26(57.8 \%)$ & 0.289 \\
\hline Thickening of intralobular septum & $50(58.1 \%)$ & $20(48.8 \%)$ & $30(66.7 \%)$ & 0.093 \\
\hline Fibrosis & $42(48.8 \%)$ & $21(51.2 \%)$ & $21(46.7 \%)$ & 0.673 \\
\hline Thickening of small vessels & $79(91.9 \%)$ & $36(87.8 \%)$ & $43(95.6 \%)$ & 0.251 \\
\hline Air bronchogram & $68(79.1 \%)$ & $30(73.2 \%)$ & $38(84.4 \%)$ & 0.199 \\
\hline Crazy-paving pattern & $41(47.7 \%)$ & $21(51.2 \%)$ & $20(44.4 \%)$ & 0.530 \\
\hline Thickening of bronchovascular bundles & $61(70.9 \%)$ & $26(63.4 \%)$ & $35(77.8 \%)$ & 0.143 \\
\hline Cavity & $4(4.7 \%)$ & $3(7.3 \%)$ & $1(2.2 \%)$ & 0.344 \\
\hline Thickening pleura & $74(86 \%)$ & $34(82.9 \%)$ & $40(88.9 \%)$ & 0.425 \\
\hline Shifting of pleura & $51(59.3 \%)$ & $21(51.2 \%)$ & $30(66.7 \%)$ & 0.145 \\
\hline Sparing subpleural region & $16(18.6 \%)$ & $9(22 \%)$ & $7(15.6 \%)$ & 0.447 \\
\hline Pleural effusion & $10(11.6 \%)$ & $6(14.6 \%)$ & $4(8.9 \%)$ & 0.508 \\
\hline Lymphadenopathy & $3(3.5 \%)$ & $0(0 \%)$ & $3(6.7 \%)$ & 0.243 \\
\hline
\end{tabular}

Data are median (IQR), $n(\%)$, or $\mathrm{n} / \mathrm{N}(\%)$, where $N$ is the total number of patients with available data. $p$ values comparing common pneumonia and severe, critical pneumonia are from $\chi^{2}$ test, Fisher's exact test, or Mann-Whitney $U$ test. $p<0.05$ has statistically significance

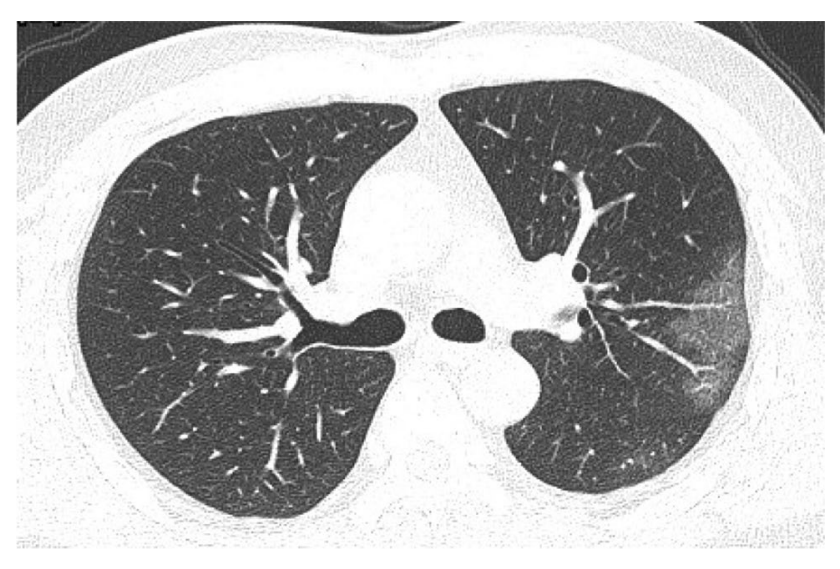

Fig. 1 Male, 41 years, initial CT of moderate category of COVID-19. Fever, cough, normal WBC, and decreased lymphocyte count. GGO with thickening of the small vessels were found in the left upper lobe to have underlying comorbidities; and the male was predominant, but no difference was found between the male and female.

Clinical symptoms are not consistent with the CT findings. Some asymptomatic patients show typical viral pneumonia features and positive RT-PCR result, who are also the infection source [6]. All the present cohort complained of clinical symptoms, such as fever, cough, or sore throat; but no difference was found in symptoms of the different severity of COVID-19. No difference was found in the ratio of normal WBC between the two groups, while the higher ratio of normal lymphocyte was found in the mild and moderate group. Decreased lymphocyte count was observed in $52 / 86(60.5 \%)$ patients. And the lower of lymphocyte count are more likely to develop into severe or critical category (Table $1,36.6 \%$ vs. $82.2 \% ; p<0.001$ ), which is similar to previous study [4]. 


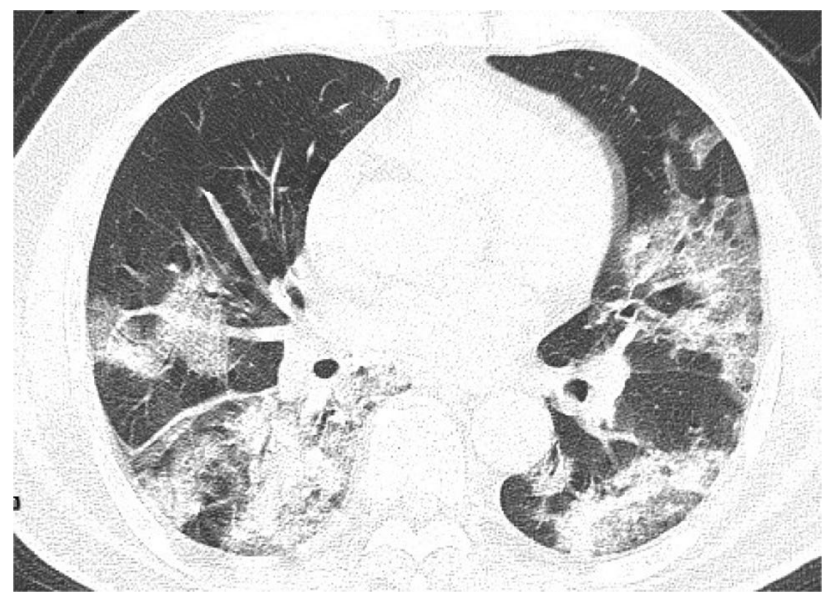

Fig. 2 Male, 68 years, initial CT of severe category of COVID-19. Fever, cough, normal WBC, and decreased lymphocyte count. Multiple GGO with consolidation, crazy-paving sign, thickening of pleural was found

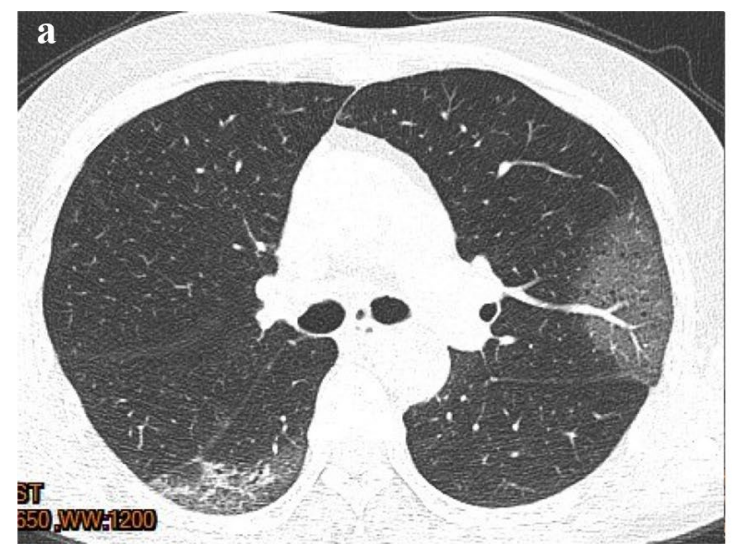

Fig. 3 Female, 62 years, initial CT of moderate category of COVID19. Fever, cough, normal WBC, and decreased lymphocyte count. a Multiple wedge-shaped GGO, consolidation, thickening of small ves-
According to the 6th version proposal issued by China; the mild category showed no sign of pneumonia [7]; there were two cases $(2 / 86,2.3 \%)$ without pneumonia in present study. The presence and severity of pneumonia is also not consistent with the clinical stages. It has been reported $5.2 \%$ severe cases without pneumonia [8]; however, all the severe or critical cases in the present study showed pneumonia. COVID-19 CT findings vary with the patient's age, immunity status, disease stage, underlying diseases and drug interventions at the time of scanning [9]. In this study, the clinical category was based on the clinical features, progression and prognosis; only the initial CT images were analyzed to evaluate the predictive value for the progression of initial CT images. The multiple lesions and lower lobe predominance were similar to the previous studies [10]. The involvement of upper lobes was more marked in the severe and critical category, suggesting the upper lobe involvement was more prone to progress into severe and critical category. GGO,

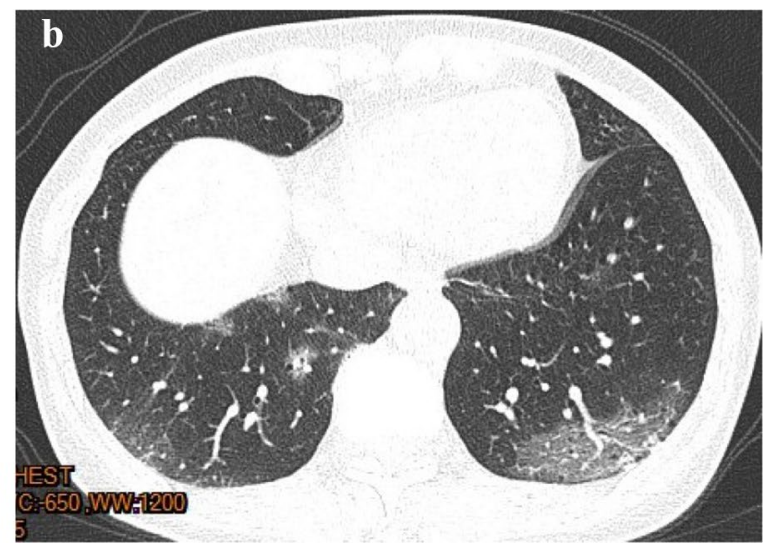

sels and air containing space. b Multiple small patchy GGO, thickening of small vessels and fibrosis
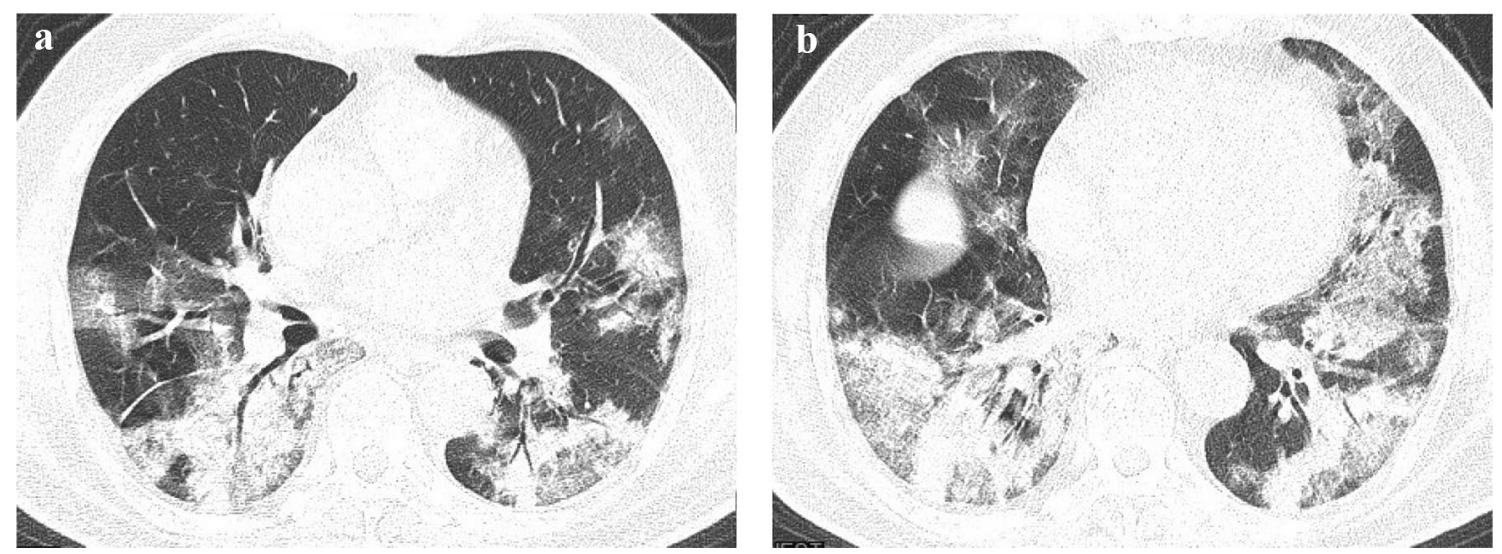

Fig. 4 Male, 64 years, initial CT of severe category of COVID-19. Fever, cough, normal WBC, and decreased lymphocyte count. a and b Showed multiple GGO with consolidation, air bronchogram, crazy-paving sign and thickening of right oblique fissure 


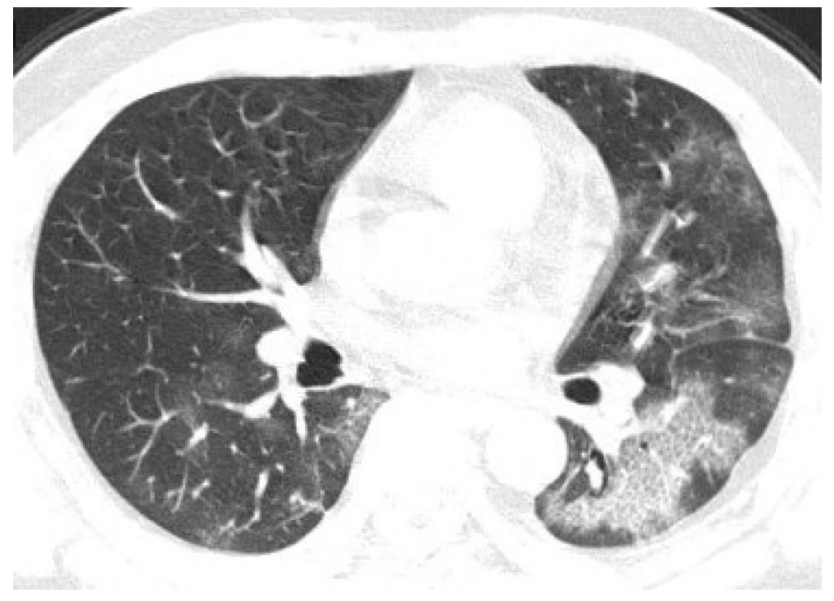

Fig. 5 Male, 41 years, initial CT of severe category of COVID-19. Fever, cough, normal WBC, and decreased lymphocyte count. Multiple GGO, crazy-paving sign, thickening of left oblique fissure

thickening of intralobular septum, thickening of small vessels, air bronchogram, crazy-paving pattern, thickening of bronchovascular bundles and thickening pleura were common features in the present cohort, which was similar to the previous reports [11-17]. The pathological basis of GGO was the bilateral diffuse alveolar damage with cellular fibromyxoid exudate, which was the first pathological findings of COVID-19 by postmortem biopsy [18]. Although, the presence of GGO was significant different between the two groups, no imaging features were the important indicators for the severe and critical category by binary logistic regression analysis, while only the decreased lymphocyte count was predictor, which may be related to a non-prognostic role of $\mathrm{CT}$ in the first period (0-5 days) and the lack of specificity of initial CT findings [19]. Moreover, the majority of the imaging features between the two groups were not different significantly. The dynamic CT imaging changes may be predictive for the severity of COVID-19.

There are several limitations in this study. First, only the first CT images were evaluated, some of them were lack of specificity in the early stage. Second, the follow up CT images were not evaluated, which may be more predictive in the evaluation of severity of COVID-19. Third, some patients had received therapy before CT scanning due to the very difficult period, which would affect the imaging manifestations. Fourth, we did not consider the patient's comorbidities.

In conclusion, the initial CT features of the different clinical category overlapped. Combining with laboratory test, especially the lymphocyte count, could help to predict the severity of COVID-19.

Supplementary Information The online version contains supplementary material available at https://doi.org/10.1007/s42058-021-00056-4.
Funding This work was financially supported by the National Natural Science Foundation of China, Grant numbers: 81871321, 81930049. Key Technologies Research and Development Program (CN), Grant number: 2016YFE0103000. National Key R\&D Program of China, Grant number: 2017YFC1308703.

\section{Compliance with ethical standards}

Conflict of interest The authors declared that they have no conflict of interest.

\section{References}

1. WHO. https://experience.arcgis.com/experience/685d0ace52 1648f8a5beeeee1b9125cd.. Accessed 21 Mar 2020.

2. Diagnosis and treatment protocols of COVID-19 infection (Trial Version 7). The national health commission of the People's Republic of China [EB/OL]. http://www.nhc.gov.cn/yzygj /s7653p/202002/8334a8326dd94d329df351d7da8aefc2.shtml.

3. Fan L, Li D, Xue H, et al. Progress and prospect on imaging diagnosis of COVID-19. Chin J Acad Radiol. 2020. https://doi. org/10.1007/s42058-020-00031-5.

4. Francone M, Iafrate F, Masci GM, et al. Chest CT score in COVID-19 patients: correlation with disease severity and shortterm prognosis. Eur Radiol. 2020. https://doi.org/10.1007/s0033 0-020-07033-y.

5. Wang D, Hu B, Hu C, et al. Clinical characteristics of 138 hospitalized patients with 2019 novel coronavirus-infected pneumonia in Wuhan. China JAMA. 2020. https://doi.org/10.1001/ jama.2020.1585.

6. Bai Y, Yao L, Wei T, et al. Presumed Asymptomatic Carrier Transmission of COVID-19. JAMA. 2020. https://doi. org/10.1001/jama.2020.25657.

7. Diagnosis and treatment protocols of COVID-19 infection (Trial Version 6). The national health commission of the People's Republic of China [EB/OL]. http://www.nhc.gov.cn/yzygj /s7653p/202002/8334a8326dd94d329df351d7da8aefc2.shtml. Accessed 18 Feb 2020.

8. Guan WJ, Ni ZY, Hu Y, et al. Clinical characteristics of 2019 novel coronavirus infection in China. MedRxiv. 2020. https:// doi.org/10.1101/2020.02.06.20020974.

9. Jin $\mathrm{YH}$, Cai L, Cheng ZS, et al. A rapid advice guideline for the diagnosis and treatment of 2019 novel coronavirus (2019$\mathrm{nCoV})$ infected pneumonia (standard version). Milit Med Res. 2020;7(1):4. https://doi.org/10.1186/s40779-020-0233-6.

10. Song F, Shi N, Shan F, et al. Emerging coronavirus 2019-nCoV pneumonia. Radiology. 2020. https://doi.org/10.1148/radio 1.2020200274.

11. Huang LHR, Yu P, et al. The correlation between clinical characteristics and CT findings in different clinical stage in COVID-19. Chin J Radiol. 2020;54(00):E003-E003. https:// doi.org/10.3760/cma.j.issn.1005-1201.2020.0003 (In Chinese).

12. Liu P, Tan X-z. Novel coronavirus (2019-nCoV) pneumonia. Radiology. 2019;2020:200257. https://doi.org/10.1148/radio 1.2020200257.

13. Kanne JP. Chest CT findings in 2019 novel coronavirus (2019$\mathrm{nCoV}$ ) infections from Wuhan, China: key points for the radiologist. Radiology. 2019;2020:200241. https://doi.org/10.1148/ radiol.2020200241.

14. Xie X, Zhong Z, Zhao W, et al. Chest CT for typical 2019-nCoV pneumonia: relationship to negative RT-PCR testing. Radiology. 2020. https://doi.org/10.1148/radiol.2020200343. 
15. Kong W, Agarwal PP. Chest imaging appearance of COVID-19 infection. Radiol Cardiothorac Imag. 2020;2(1):e200028. https:// doi.org/10.1148/ryct.2020200028.

16. Li X, Zeng X, Liu B, et al. COVID-19 infection presenting with CT Halo Sign. Radiol Cardiothorac Imag. 2020;2(1):e200026. https://doi.org/10.1148/ryct.2020200026.

17. Fang $\mathrm{Y}, \mathrm{Zhang} \mathrm{H}, \mathrm{Xu} \mathrm{Y}$, et al. CT manifestations of two cases of 2019 novel coronavirus (2019-nCoV) pneumonia. Radiology. 2019;2020:200280. https://doi.org/10.1148/radiol.2020200280.

18. Xu Z, Shi L, Wang Y, et al. Pathological findings of COVID19 associated with acute respiratory distress syndrome.
Lancet Respirat Med. 2020. https://doi.org/10.1016/S2213 $-2600(20) 30076-X$

19. Li L, Yang L, Gui S, et al. Association of clinical and radiographic findings with the outcomes of 93 patients with COVID-19 in Wuhan. China Theranostics. 2020;10(14):6113-21.

Publisher's Note Springer Nature remains neutral with regard to jurisdictional claims in published maps and institutional affiliations. 